\title{
MÉTHODE DE COMPTAGE AUTOMATIQUE DES GRAINS DE POLLEN : ÉTUDE DE LA PRODUCTION POLLINIQUE CHEZ LE TOURNESOL (HELIANTHUS ANNUUS L., COMPOSITAE)
}

\author{
Y. LOUBLIER *, Ph. DOUAULT et M.H. PHAM-DELEGUE \\ Laboratoire de Neurobiologie comparée des Invertébrés \\ I.N.R.A.-C.N.R.S., 91440 Bures-sur-Yvette
}

\begin{abstract}
RÉSUMÉ
Les auteurs décrivent une méthode de comptage automatique des grains de pollen en utilisant un compteur de particules «Coulter Counter». Cette technique permet la quantification rapide de la production pollinique chez les végétaux assurant le traitement d'un grand nombre d'échantillons ainsi que plusieurs répétitions par mesure mettant en évidence la bonne reproductibilité de la méthode. Le pollen de tournesol (Helianthus annuus L.) a été utilisé pour mettre au point la méthode de comptage.
\end{abstract}

\section{INTRODUCTION}

Le problème de la pollinisation entomophile a pris récemment une acuité particulière, notamment en raison du développement de programmes d'amélioration végétale fondés sur la création de variétés hybrides. C'est pourquoi les paramètres d'attraction des insectes pollinisateurs, tels que l'abeille domestique, doivent être pris en compte en vue d'une pollinisation contrôlée.

Chez l'abeille domestique, l'intensité de butinage est liée à la mise en jeu d'événements sensoriels successifs conduisant à la mise en place d'un apprentissage (MAsson, 1982) : tout en transférant le pollen des anthères aux stigmates, les butineuses associent les caractéristiques visuelles (forme, couleur) et olfactives (arômes) de la plante à ses caractéristiques alimentaires (quantité et qualité de l'approvisonnement en nectar et pollen). Ce processus de mémorisation au cours de la prise alimentaire induit ultérieurement une orientation sélective des butineuses vers la plante, qui joue un rôle déterminant dans leur efficacité pollinisatrice. Parmi les paramètres de renforcement alimentaire, les pollens constituent le principal apport protéique ; ils peuvent de plus constituer une source d'émissions chimiques intervenant comme signal d'orientation pour les butineuses. Ainsi, en

* Adresse actuelle : Station de Zoologie et d'Apidologie, I.N.R.A., 84140 Montfavet. 
préalable à une étude biochimique des pollens en tant que facteurs de choix pour l'abeille, il nous est apparu nécessaire de disposer d'une méthode d'estimation des quantités de pollens disponibles sur la plante. Dans ce but la mise au point d'une technique fiable et rapide a été réalisée, sur un modèle de plante à pollinisation strictement entomophile, le tournesol Helianthus annuus $\mathbf{L}$.

Les méthodes d'évaluation de la production pollinique chez plusieurs espèces végétales ont fait appel jusqu'ici à différentes techniques décrites par plusieurs auteurs : Oberle et Goertzen (1952), Obolensky (1958), Sree Rangasamy et Raman (1973), Sapra et Hughes (1975), Segala et al. (1980), Traynor (1981), Nikovitz et al. (1983), Szabo (1985). Celles-ci consistaient en une dilacération des étamines dans un liquide dispersant suivie d'une numération des grains de pollen libérés, au moyen d'un hématimètre.

D'autres auteurs, tels que Warakomska (1972) et SimidTchev (1977), ont procédé par pesées d'anthères, les résultats étant alors fournis en masse de pollen produite par fleur.

Enfin, Cour (1974), MahHou (1981) et MaAmar (1983) ont pratiqué une attaque chimique des fleurs suivie d'une analyse volumétrique fractionnelle.

Les méthodes employées par ces différents auteurs nécessitent un temps de manipulation et d'analyse microscopique important. Le présent travail permet de diminuer le temps de traitement des fleurs et de soumettre au comptage la totalité du matériel pollinique.

Les avantages de rapidité d'exécution et de bonne reproductibilité de cette méthođe rendent possible le traitement d'un plus grand nombre d'échantillons, permettant d'établir des comparaisons fiables.

\section{Matériel végétal}

\section{MATERIEL ET METHODES}

Le matériel végétal provient de fleurons de tournesol (Helianthus annuus L.) prélevés sur différents hybrides et lignées mâles fertiles mis à notre disposition par la Station d'Amélioration des Plantes de l'I.N.R.A. (Clermont-Ferrand).

Le prélèvement des échantillons a été effectué au stade II de la floraison du capitule (50\% des fleurons tubulés matures mais non déhiscents), à raison de 10 fleurons par capitule, et répété sur 5 capitules par génotype. L'ensemble des échantillons est ensuite conservé à la température de $-18^{\circ} \mathrm{C}$.

\section{Méthode}

Les étapes de traitement appliqué aux échantillons sont les suivantes :

1) Dix fleurons d'un capitule sont déposés dans un bécher et écrasés à l'aide d'un agitateur en verre à bout rôdé dans quelques gouttes d'eau distillée. Dix millilitres d'acide sulfurique pur 
sont ensuite versés sur les fleurons. Une macération de vingt-quatre heures est nécessaire pour désagréger les tissus végétaux du fleuron sans endommager les grains de pollen particulièrement résistants des Hélianthus.

2) Le mélange est ensuite dilué dans quatre-vingt-dix millilitres d'eau distillée contenant deux pour cent de «Triton» [agent mouillant utilisable en milieu acide et permettant la dispersion des particules (CAMBon, 1981)]. La totalité du mélange est centrifugée pendant sept minutes à trois mille tours par minute (sept cents $\mathrm{g}$ ) dans des tubes à fond conique de 50 millilitres afin d'obtenir un culot. On rince ensuite deux fois à l'eau distillée.

3) Sur le culot obtenu, on pratique une acétolyse selon la méthode de ERdTMan (1960). Deux centrifugations dans l'eau distillée sont ensuite nécessaires pour rincer le culot.

4) Pour procéder à la séparation des grains de pollen entre eux et pour éliminer les particules végétales, nous avons utilisé un générateur d'ultra sons BRANSONIC modèle 32 FTZ, monofréquentiel, de cinquante kilocycles par seconde. Le culot est mis en suspension dans cinq millilitres d'alcool à $95^{\circ}$ et déposé dans la cuve jusqu'à ce que les trois quarts du tube soient immergés. Les meilleurs résultats obtenus pour l'élimination des grosses particules végétales correspondent à un temps d'exposition de quinze minutes. On constate ainsi une très bonne dispersion des grains de pollen, de même qu'une absence de leur fracturation.

5) Après centrifugation, l'alcool est éliminé et le culot est alors repris dans cinquante millilitres d'un électrolyte approprié : «l'Isoton». Cette solution saline est destinée au comptage et permet de pratiquer les mesures successives.

Pour effectuer le comptage des grains de pollen, nous avons utilisé un compteur de particules «Coulter Counter» ZB Coultronics équipé d'un tube à électrodes de platine muni d'un orifice de deux cent quatre-vingt microns.

Le principe de comptage automatique repose sur le fait que toutes particules en mouvement dans un liquide déplacent un certain volume proportionnel à leur taille. Ces variations de volume ainsi engendrées sont amplifiées électroniquement, étalonnées à l'intérieur de la classe de taille choisie et comptabilisées sous forme d'impulsions.

L'appareil est réglé pour enregistrer des déplacements de volumes correspondant à des particules de vingt cinq à trente cinq microns de diamètre. La quantité minimale d'électrolyte devant passer au travers de l'ouverture du tube pour permettre le comptage est de deux millilitres.

Les contraintes techniques de l'appareil imposent une séparation des particules, assurée par le passage aux ultra sons, et une concentration minimale de l'ordre de cinq mille particules par millilitre; en traitant dix fleurons par capitule, la concentration utilisée est toujours supérieure à celle-ci. Le volume total de solution permet de pratiquer quatre mesures successives pour un même échantillon, chaque mesure étant faite après homogénéisation de la solution. Pour chaque lignée mâle fertile et hybride une vérification au microscope a été effectuée selon la méthode de Cour et Loublier (1980) afin d'observer l'état des grains de pollen soumis aux ultrasons (Dumait, 1962 ; MCINTYRe et Norris, 1964 ; MarCeAU, 1969 ; Cerceau et al., 1970. Cette observation permet également de repérer la présence de particules végétales et de pollens de contamination susceptibles d'être comptés comme appartenant au tournesol.

Nous avons donc répertorié :

a) Le nombre de grains de pollen de tournesol : entiers, fissurés et cassés.

b) Le nombre d'endexines isolées.

c) Le nombre de particules végétales et de grains de pollen de contamination dont la taille est comparable à celle du pollen de tournesol. 
TABL. 1. - Evaluation de la production pollinique du tournesol (Clermont-Ferrand, 1984) obtenue par comptage au «Coulter-Counter》

TABL. 1. - Evaluation of sunflower pollen production (samples from Clermont-Ferrand, 1984) using $a$ «Coulter-Counter》

\begin{tabular}{|c|c|c|c|c|c|c|c|}
\hline & & A & $B$ & $\mathrm{C}$ & D & $\mathrm{E}$ & $\mathbf{M}$ \\
\hline$\sigma^{\pi}$ & PAH3 & $\begin{array}{c}395181 \\
(1,33)\end{array}$ & $\begin{array}{c}366913 \\
(0,93)\end{array}$ & $\begin{array}{c}356931 \\
(1,05)\end{array}$ & $\begin{array}{c}377356 \\
(1,03)\end{array}$ & $\begin{array}{c}400031 \\
(0,42)\end{array}$ & $\begin{array}{c}379282 \\
(4,82)\end{array}$ \\
\hline H & $\mathrm{H} 4 \mathrm{P} 1$ & $\begin{array}{c}429175 \\
(1,85)\end{array}$ & $\begin{array}{c}322906 \\
(0,84)\end{array}$ & $\begin{array}{c}379131 \\
(2,20)\end{array}$ & $\begin{array}{c}432313 \\
(0,53)\end{array}$ & $\begin{array}{c}441800 \\
(1,12)\end{array}$ & $\begin{array}{l}401065 \\
(12,48)\end{array}$ \\
\hline$\sigma^{\pi}$ & RHA273 & $\begin{array}{c}324631 \\
(0,12)\end{array}$ & $\begin{array}{c}351056 \\
(1,12)\end{array}$ & $\begin{array}{c}314431 \\
(0,94)\end{array}$ & $\begin{array}{c}327013 \\
(1,80)\end{array}$ & $\begin{array}{c}340063 \\
(0,70)\end{array}$ & $\begin{array}{c}331439 \\
(4,31)\end{array}$ \\
\hline H & PARIOU & $\begin{array}{c}371525 \\
(2,70)\end{array}$ & $\begin{array}{c}324919 \\
(2,73)\end{array}$ & $\begin{array}{c}287231 \\
(5,30)\end{array}$ & $\begin{array}{c}363694 \\
(1,75)\end{array}$ & $\begin{array}{c}368575 \\
(1,04)\end{array}$ & $\begin{array}{l}343189 \\
(10,64)\end{array}$ \\
\hline$\sigma^{\pi}$ & RHA274 & $\begin{array}{c}385950 \\
(1,38)\end{array}$ & $\begin{array}{c}361263 \\
(2,09)\end{array}$ & $\begin{array}{c}364313 \\
(2,27)\end{array}$ & $\begin{array}{c}369313 \\
(1,68)\end{array}$ & $\begin{array}{c}361906 \\
(0,33)\end{array}$ & $\begin{array}{c}367549 \\
(2,82)\end{array}$ \\
\hline $\mathrm{H}$ & RODEO & $\begin{array}{c}307763 \\
(1,32)\end{array}$ & $\begin{array}{c}331900 \\
(0,57)\end{array}$ & $\begin{array}{c}401563 \\
(0,81)\end{array}$ & $\begin{array}{l}329344 \\
(1,24)\end{array}$ & $\begin{array}{c}353456 \\
(1,33)\end{array}$ & $\begin{array}{c}346805 \\
(10,52)\end{array}$ \\
\hline $\mathbf{H}$ & MIRASOL & $\begin{array}{r}343813 \\
(0,69) \\
\end{array}$ & $\begin{array}{c}377606 \\
(0,55)\end{array}$ & $\begin{array}{c}352044 \\
(0,63) \\
\end{array}$ & $\begin{array}{c}358194 \\
(1,23) \\
\end{array}$ & $\begin{array}{c}373075 \\
(1,34) \\
\end{array}$ & $\begin{array}{c}350,946 \\
(3,93)\end{array}$ \\
\hline$\sigma^{\pi}$ & $\mathrm{ZN} 41$ & $\begin{array}{c}324100 \\
(1,79)\end{array}$ & $\begin{array}{c}319494 \\
(3,84)\end{array}$ & $\begin{array}{c}335200 \\
(1,14)\end{array}$ & $\begin{array}{c}344069 \\
(1,33)\end{array}$ & $\begin{array}{c}381969 \\
(1,10)\end{array}$ & $\begin{array}{c}340966 \\
(7,29)\end{array}$ \\
\hline $\mathrm{H}$ & BOLERO & $\begin{array}{c}377444 \\
(1,99) \\
\end{array}$ & $\begin{array}{c}379781 \\
(1,14) \\
\end{array}$ & $\begin{array}{c}366938 \\
(2,03) \\
\end{array}$ & $\begin{array}{c}390244 \\
(1,50) \\
\end{array}$ & $\begin{array}{c}388394 \\
(1,78) \\
\end{array}$ & $\begin{array}{c}380560 \\
(2,46)\end{array}$ \\
\hline$\sigma^{x}$ & PAC2 & $\begin{array}{c}355619 \\
(0,82)\end{array}$ & $\begin{array}{c}376 \overline{769} \\
(1,15)\end{array}$ & $\begin{array}{c}391538 \\
(3,74)\end{array}$ & $\begin{array}{c}371819 \\
(1,72)\end{array}$ & $\begin{array}{c}394413 \\
(0,75)\end{array}$ & $\begin{array}{c}378032 \\
(4,17)\end{array}$ \\
\hline $\mathbf{H}$ & $\mathrm{H} 4 \mathrm{P} 2$ & $\begin{array}{c}388500 \\
(2,20)\end{array}$ & $\begin{array}{c}349813 \\
(0,85) \\
\end{array}$ & $\begin{array}{c}400269 \\
(1,53) \\
\end{array}$ & $\begin{array}{c}41.6269 \\
(1,02) \\
\end{array}$ & $\begin{array}{c}326875 \\
(1,31)\end{array}$ & $\begin{array}{c}376345 \\
(9,82) \\
\end{array}$ \\
\hline $0^{\pi}$ & RHA266 & $\begin{array}{c}318106 \\
(0,90)\end{array}$ & $\begin{array}{c}3720.53 \\
(1,71)\end{array}$ & $\begin{array}{c}260344 \\
(1,29)\end{array}$ & $\begin{array}{c}354625 \\
(1,12)\end{array}$ & $\begin{array}{c}292538 \\
(0,90)\end{array}$ & $\begin{array}{l}319535 \\
(14,19)\end{array}$ \\
\hline $\mathbf{H}$ & PRIMASOL & $\begin{array}{c}376631 \\
(1,08)\end{array}$ & $\begin{array}{c}337719 \\
(0,21)\end{array}$ & $\begin{array}{c}361156 \\
(1,66)\end{array}$ & $\begin{array}{c}374013 \\
(0,86)\end{array}$ & $\begin{array}{c}383719 \\
(1,36)\end{array}$ & $\begin{array}{c}365848 \\
(5,39) \\
\end{array}$ \\
\hline $0^{3}$ & $\overline{\text { RHA271 }}$ & $\begin{array}{c}45563 \\
(0,84)\end{array}$ & $\begin{array}{c}443788 \\
(3,01)\end{array}$ & $\begin{array}{c}422369 \\
(0,83)\end{array}$ & $\begin{array}{c}406700 \\
(0,58)\end{array}$ & $\begin{array}{c}438063 \\
(0,95)\end{array}$ & $\begin{array}{c}431297 \\
(3,83)\end{array}$ \\
\hline $\mathbf{H}$ & ELIA & $\begin{array}{c}413981 \\
(1,72)\end{array}$ & $\begin{array}{c}398538 \\
(0,92) \\
\end{array}$ & $\begin{array}{c}408550 \\
(0,79) \\
\end{array}$ & $\begin{array}{c}406050 \\
(0,62) \\
\end{array}$ & $\begin{array}{c}361138 \\
(0,84)\end{array}$ & $\begin{array}{c}397651 \\
(5,32)\end{array}$ \\
\hline $0^{*}$ & PR 4 & $\begin{array}{c}323.606 \\
(0,92)\end{array}$ & $\begin{array}{c}342350 \\
(2,87)\end{array}$ & $\begin{array}{c}314631 \\
(0,91)\end{array}$ & $\begin{array}{c}338775 \\
(0,52)\end{array}$ & $\begin{array}{c}306581 \\
(0,56)\end{array}$ & $\begin{array}{c}325189 \\
(4,71)\end{array}$ \\
\hline $\mathbf{H}$ & IC & $\begin{array}{c}351238 \\
(1,59)\end{array}$ & $\begin{array}{c}404300 \\
(1,08)\end{array}$ & $\begin{array}{c}347800 \\
(1,91)\end{array}$ & $\begin{array}{c}379956 \\
(1,06)\end{array}$ & $\begin{array}{c}383969 \\
(1,55)\end{array}$ & $\begin{array}{c}373453 \\
(6,36) \\
\end{array}$ \\
\hline $0^{\pi}$ & BZA2 & $\begin{array}{c}363200 \\
(1,14)\end{array}$ & $\begin{array}{c}270419 \\
(0,67)\end{array}$ & $\begin{array}{c}300931 \\
(1.63)\end{array}$ & $\begin{array}{c}325031 \\
(3,08)\end{array}$ & $\begin{array}{c}279088 \\
(1,31)\end{array}$ & $\begin{array}{l}307734 \\
(12,20)\end{array}$ \\
\hline $\mathrm{H}$ & MARIANNE & $\begin{array}{c}247600 \\
(1,04) \\
\end{array}$ & $\begin{array}{c}329131 \\
(0,70)\end{array}$ & $\begin{array}{c}266138 \\
(0,92)\end{array}$ & $\begin{array}{c}346244 \\
(1,04) \\
\end{array}$ & $\begin{array}{c}285831 \\
(1,43) \\
\end{array}$ & $\begin{array}{r}294989 \\
(14,14) \\
\end{array}$ \\
\hline$\sigma^{\pi}$ & \multicolumn{7}{|c|}{$\begin{array}{l}\text { Lignée o fertile. } \\
\text { Male-fertile line. }\end{array}$} \\
\hline $\mathbf{H}$ & \multicolumn{7}{|c|}{$\begin{array}{l}\text { Hybride correspondant. } \\
\text { Corresponding hybrid. }\end{array}$} \\
\hline \multicolumn{8}{|c|}{ A, B, C, D, E : 5 capitules différents/génotype. } \\
\hline $\mathbf{M}$ & \multicolumn{7}{|c|}{$\begin{array}{l}\text { Moyenne des } 5 \text { capitules. } \\
\text { Mean of } 5 \text { heads. }\end{array}$} \\
\hline () & \multicolumn{7}{|c|}{$\begin{array}{l}\text { Ecart-type. } \\
\text { Standard deviation. }\end{array}$} \\
\hline
\end{tabular}




\section{RESULTATS}

Nous avons pu, par la méthode décrite, traiter quatre-vingt-quinze échantillons, avec quatre répétitions par échantillon (neuf lignées mâles fertiles et 10 hybrides correspondant, à raison de cinq capitules par génotype).

Pour tous les génotypes étudiés (cf. tableau 1), les valeurs moyennes de production pour dix fleurons sont comprises entre 294989 (hybride MARIANNE) et 431297 grains de pollen (lignée RHA 271, parent mâle de ELIA).

En ce qui concerne la reproductibilité des mesures, les coefficients de variation, pour quatre mesures par échantillon, varient de 0,12\% (lignée RHA 273, parent mâle de PARIOU) à 5,30\% (hybride PARIOU) tandis que pour la reproductibilité inter capitule, pour cinq capitules par génotype, les coefficients de variation s'échelonnent entre 2,82\% (lignée RHA 274, parent mâle de RODEO et MIRASOL) et 14,19 \% (lignée RHA 266, parent mâle de PRIMASOL).

La lecture de ce tableau fait ressortir :

- un faible taux de variation inter mesures, ce qui nous permet d'envisager, pour des études ultérieures, de procéder à un nombre inféreur de répétitions par échantillon ;

- des différences génotypiques dans la production pollinique du tournesol. Une étude quantifiée de ces différences fait l'objet d'un travail en cours;

- un coefficient de variation inter-capitules parfois élevé (supérieur à dix pour cent), qui est l'indice d'une hétérogénéité dans la production pollinique de certains génotypes. Le comptage automatique des grains de pollen nous autorise à envisager pour l'avenir d'effectuer des prélèvements sur un plus grand nombre de capitules de façon à mettre en évidence l'origine génétique possible de ces variations. Les analyses miscroscopiques ont montré que (cf. tableau 2) :

- sur l'ensemble des particules comptées à chaque mesure, les grains de pollen de tournesol représentaient entre 97,73\% (hybride PARIOU) et 99,25\% (lignée RHA 271, parent mâle de ELIA);

- les ultra sons n'engendrent que très peu d'artéfacts. Le taux de pollens cassés varie de $0,02 \%$ (lignée BZA 2, parent mâle de MARIANNE) à $0,14 \%$ (lignée PAC2, parent mâle de H4P2). Celui des endexines isolées de $0 \%$ à $0,45 \%$ (lignée PAH3, parent mâle de H4P1) ;

- les pourcentages de particules végétales de la taille d'un grain de pollen varient de 0,54 \% (lignée ZN41, parent mâle de BOLERO) à 1,75 \% (lignée PR4, parent mâle de 1C). 


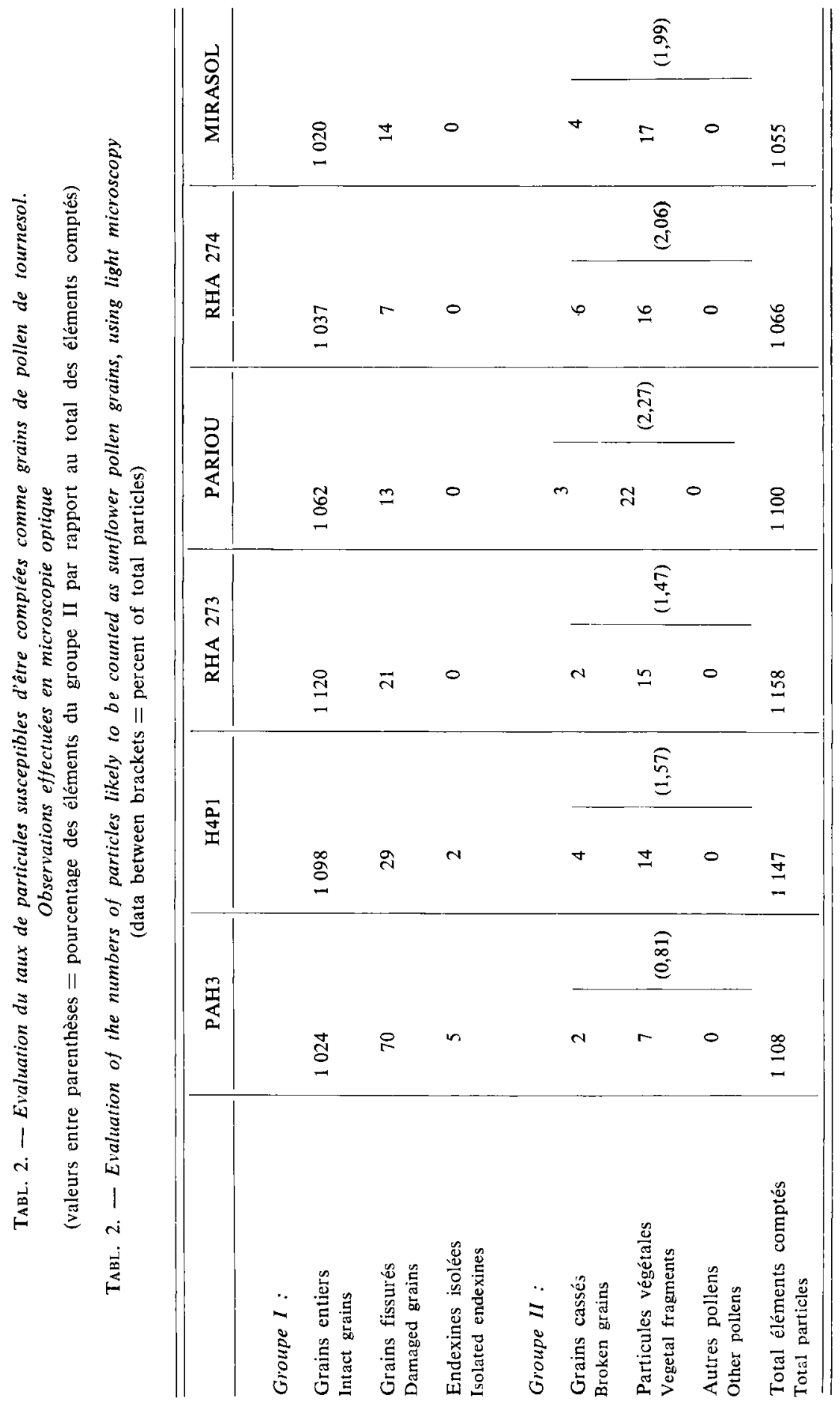




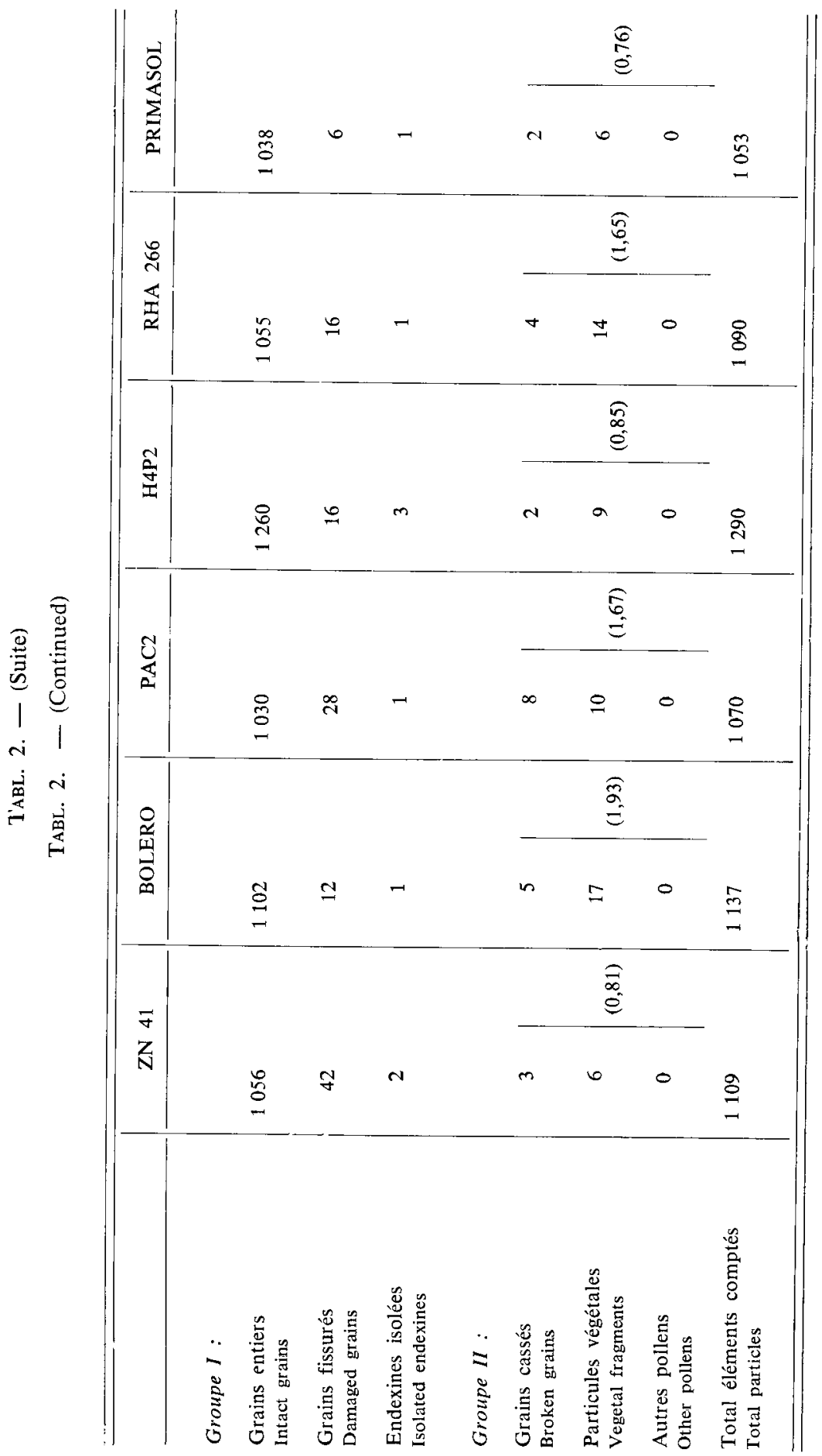




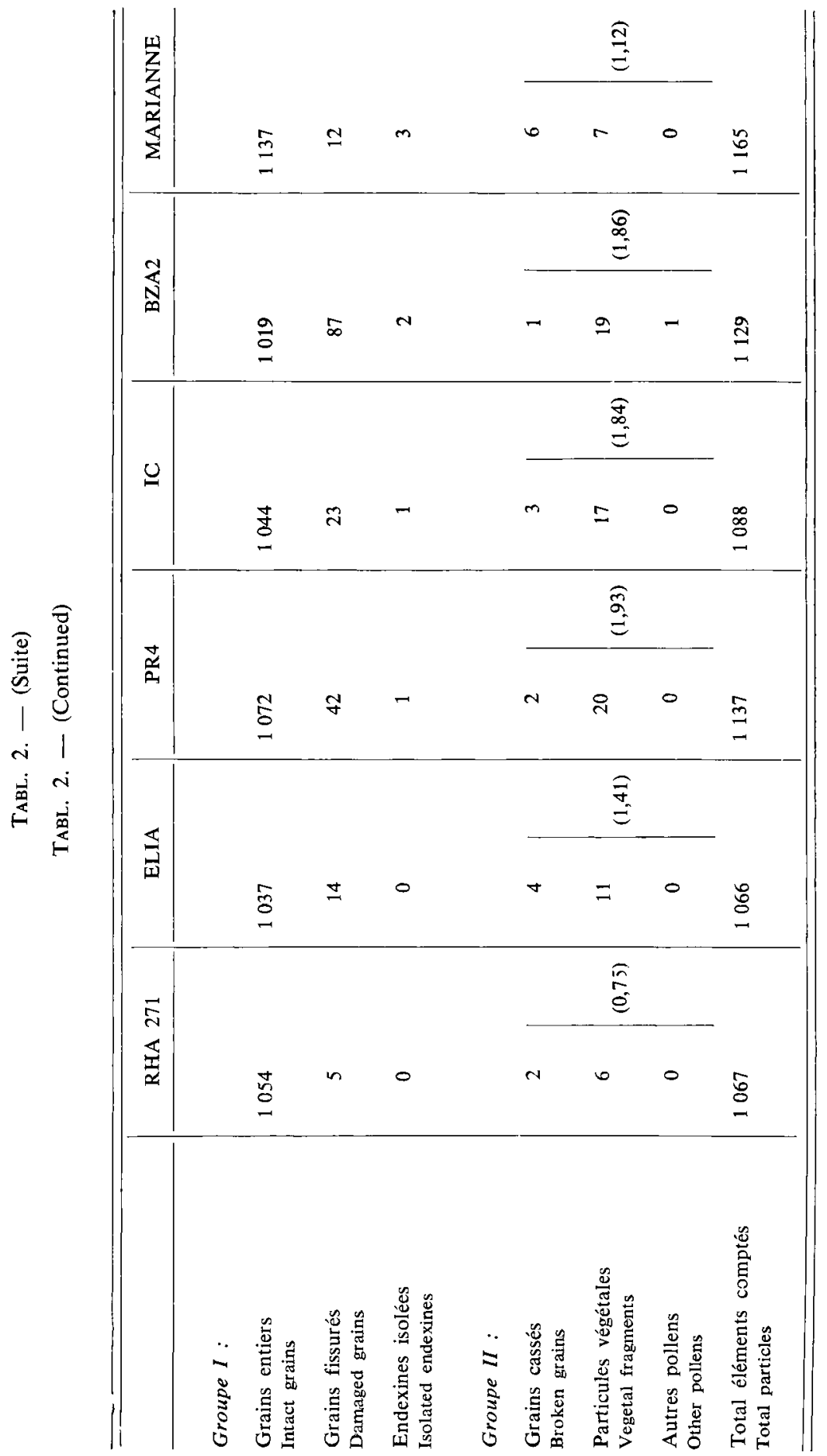




\section{CONCLUSION}

La méthode décrite ici présente plusieurs avantages sur celles utilisées auparavant. En particulier :

L'introduction des ultra sons après le traitement des grains de pollen par l'acétolyse permet :

- de réduire à vingt-quatre heures l'action de l'acide sulfurique sur les fleurs (MAHHOU, 1981; MAamar, 1983);

- d'éliminer le passage à la potasse et les filtrations sur tamis (MaHHOU et MaAmar, op. cit.);

- de dissocier les grains de pollen entre eux, favorisant une bonne homogénéisation des solutions de comptage;

- de réduire au maximum le nombre d'impuretés qui serait nuisible au bon déroulement du comptage.

L'utilisation du «Coulter Counter», par sa rapidité d'exécution et sa grande fiabilité (Edwards et Gunson, 1970; Stockmarr, 1971) rend possible le traitement d'un plus grand nombre d'échantillons.

Cette méthode mise au point sur le tournesol met en évidence des différences dans la production pollinique selon les génotypes mais aussi pour un même génotype des variations relativement importantes entre capitules. Son application à un plus grand nombre de génotypes et de capitules est en cours afin de mieux cerner l'aspect génétique de ces variations.

Enfin, soulignons qu'il est possible d'adapter cette méthode de comptage à d'autres végétaux. Toutefois, il sera nécessaire de déterminer au préalable, le nombre de fleurs à prélever afin de se placer dans les conditions optimales d'utilisation du «Coulter Counter», et de vérifier l'état des exines des grains de pollen soumises aux ultra sons, la résistance des grains variant en fonction de leur morphologie (MCINTYRE et NorRIS, 1964).

Ainsi, grâce à la méthode décrite, il est possible d'envisager l'étude comparée des productions polliniques de différentes espèces végétales ou de différentes lignées ou variétés d'une même espèce. De telles études pourraient permettre d'apprécier d'une part le déterminisme génétique de la production pollinique d'un végétal, l'héritabilité de ce caractère et d'autre part l'incidence de ce potentiel pollinique sur les choix des insectes pollinisateurs.

Reçu pour publication en mars 1986. 


\section{REMERCIEMENTS}

Nous remercions M. G. RiBa de la Station de Recherches de Lutte Biologique de la Minière (I.N.R.A.) d'avoir mis à notre disposition son compteur de particules.

\section{SUMMARY \\ AUTOMATIC METHOD FOR COUNTING POLLEN GRAINS : A STUDY OF SUNFLOWER POLLEN PRODUCTION (HELIANTHUS ANNUUS L., COMPOSITAE)}

A convenient method for counting pollen grains to evaluate sunflower pollen production is presented. Following acetolysis and ultrasonic treatment, pollen grains are counted with a « Coulter Counter ».

Mode of execution :

1) 10 mature, non-dehiscent florets are put in a laboratory vessel and ground in a few drops of distilled water in a glass homogenizer. Then $10 \mathrm{ml}$ pure sulfuric acid are added to act on the substratum for 24 hours.

2) $90 \mathrm{ml}$ of a $2 \%$ aqueous solution of triton are added and centrifuged until one single sediment is obtained. Each centrifugation is done for $7 \mathrm{~min}$. at $3000 \mathrm{rpm}$ (about $700 \mathrm{~g}$ ).

3) Acetolysis is conducted following the method of ERDTMAN (1960).

4) The sediment is dissolved in $5 \mathrm{ml}$ ethanol at $95^{\circ} \mathrm{C}$ and subjected for $15 \mathrm{~min}$. to a ultrasonic treatment with frequency of 50 kilocycles/sec.

5) The particles (pollen) are reconcentrated by centrifugation and rediluted in $50 \mathrm{ml}$ Isoton. This solution is used to make four subsequent measurements with the "Coulter Counter ».

This technique makes the rapid treatment of numerous samples with high reproducibility possible. When applied to sunflower florets, it allows the determination of pollen production differences among genotypes and among heads of a given genotype.

This counting method can also be applied to other plants; however, to avoid damage to grains, resistance to ultrasonic treatment must be previously evaluated.

\section{ZUSAMMENFASSUNG}

\section{EINE AUTOMATISCHE METHODE ZUR ZAHLUNG VON POLLENKÖRNERN : UNTERSUCHUNG DER PRODUKTION VON SONNENBLUMEN-POLLEN (HELIANTHUS ANNUUS L., COMPOSITAE)}

Es wird eine bequeme Methode zur Zählung von Pollenkörnern vorgestellt, um die Produktion von Sonnenblumen-Pollen zu bestimmen. Nach Azetolyse und Ultraschall-Behandlung werden die Pollenkörner mit einem «Coulter Counter» gezählt.

Durchführung der Methode :

1.) 10 reife, noch nicht geöffnete Einzelblüten werden in ein Becherglas gebracht und in einigen Tropfen destillierten Wassers mittels eines Rührstabes aus Glas zerrieben. Anschließend läßt man $10 \mathrm{ml}$ reiner Schwefelsäure durch $24 \mathrm{~h}$ einwirken. 
2.) Zugabe von $90 \mathrm{ml}$ einer $2 \%$ wässrigen Triton-Lösung und so lange zentrifugieren, bis man ein einziges Sediment erhält. Jede Zentrifugation erfolgt bei $3000 \mathrm{t} / \mathrm{mn}$ (etwa 700 g) durch 7 Minuten.

3.) Durchführung einer Azetolyse nach der Methode FrdTMan (1960)

4.) Auflösung des Sediments in $5 \mathrm{ml}$ Alkohol bei $95^{\circ} \mathrm{C}$ und Behandlung mit Ultraschall von einer Frequenz von $50 \mathrm{Kilozyklen} / \mathrm{sec}$. während $15 \mathrm{~min}$.

5.) Konzentrierung der Partikel (Pollen) durch Zentrifugation und Auflösung in $50 \mathrm{ml}$ Isoton. Diese Lösung wird zur Durchführung von vier aufeinanderfolgenden Messungen im «Coulter Counter » benutzt.

Diese Methode ermöglicht die schnelle Verarbeitung einer großen Pollenzahl mit hoher Reproduzierbarkeit. Angewandt auf Einzelblüten von Sonnenblumen, können Unterschiede in det Pollenproduktion zwischen Genotypen und zwischen Blütenköpfen eines bestimmten Genotyps festgestellt werden.

Diese Zählmethode kann auch auf andere Pflanzen angewandt werden; um aber Schäden an den Körnern zu vermeiden, muß vorher ihre Widerstandsfähigkeit gegenüber der Ultraschall. Behandlung geprüft werden.

\section{RÉFERENCES BIBLIOGRAPHIQUES}

Cambon G., 1981. - Relations entre le contenu pollinique de l'atmosphère et le couvert végétal en Méditerranée occidentale, à Montpellier (France), Valencia (Espagne) et Oran (Algérie). Thèse de biologie végétale, Montpellier, $105 \mathrm{p}$.

Cerceau M.T. et al., 1970. - Cassure du pollen par les ultrasons pour l'étude structurale de l'éxine au microscope électronique à balayage. C.R. Acad. Sci., Paris, t. 270, 66-69.

Cour P., 1974. - Nouvelles techniques de détection des flux et retombées polliniques. Etude de la sédimentation des pollens et des spores à la surface des sols. Pollen et Spores, 16 (1), 103-141.

Cour P., Loublier Y., 1980. - Contrôle d'identité et de pureté des lots de pollens destinés à la préparation d'extraits allergéniques à usage diagnostique ou thérapeutique. Rev. Franç. Allergol., 20 (4-5), 197-201.

Dumart P., 1962. - L'action des ultrasons sur les pollens. Note préliminaire. Pollen et Spores, 4 (1), 175-180.

Edwards K.J., Gunson A.R., 1978. - A procedure for the determination of exotic pollen concentrations with a Coulter Counter. Pollen et Spores, 20 (2), 303-309.

Erdtman G., 1960. - The acetolysis method. A revised description. In. Svesk. Bot. Tidskv., 54 (4), 561-564.

MaAmar M.M., 1983. - Contribution à la pollinisation de l'olivier (Olea europea). Capacité de production pollinique des cultivars et abondance du pollen dans l'atmosphère du verger. Thèse Doct. Ing. Agronomie, Université du Languedoc, Montpellier, 125 p.

Mahrou A., 1981. - Possibilités d'utiliser la quantité de pollen de pommier Malus pumila Mill. présente dans l'atmosphère d'un verger de la même espèce comme indice prévisionnel de rendement. D.E.A. d'Agronomie, E.N.S.A., Montpellier, 45-80.

Marceau L., 1969. - Effets sur le pollen des ultrasons de basse fréquence. Pollen et Spores, 11 (1), $147-164$. 
Masson Cl., 1982. - Physiologie sensorielle et comportement de l'abeille. C.R. Séances Acad. Agric. Fr., 17 nov., 1350-1361.

MCINTYRe D.J., Norris G., 1964. - Effect of ultrasounds on recent spores and pollens. N.Z. J. Sci., 7 (2), 242-257.

Nikovitz A. et al., 1983. - Nectar and pollen yield of sunflower varieties and hybrids of different genotypes. Int. Sunflower Conf., Szeksard (Hongrie), 24-27 juillet, 55-56.

Oberle G.D., Goertzen K.L., 1952. - A method for evaluating pollen production of fruit species. Proc. Am. Soc. Hort. Sci, 59, 263-265.

Obolensky G., 1958. - Production of pollen in different lines of sunflowers and its relation to production of crossed seed. Qual. Plant. Mater. veg., 5, 64-84.

SAPRA V.T., Hughes J.L., 1975. - Pollen production in hexaploid triticale. Euphytica, 24, 237-243.

Segala A., Segala M., Piquemal G., 1980. - Recherches en vue d'améliorer le degré d'autogamie des cultivars de tournesol (Helianthus annuus) Ann. Amélior. Plantes, 30 (2), 161-174.

Simidtchev Y., 1977. - Symposium International de la flore mellifère (Budapest, 1976), (a) 139-149, (b) 93-100, (c) 125-135, (d) 117-125.

SRe Rangasamy S.R., Raman V.S., 1973. - Pollen production in diploids and autotétraploids of rice (Oriza sativa L.). Pollen et Spores, 15 (2), 189-193.

StockmarR J., 1972. - Determination of spore concentration on electronic particle counter. Danm. Geol. Undersog. Arbog., 87-89.

Szabo T.I., 1985. - Variability of flower, nectar, pollen and seed production in some Canadian Canola (Rapeseed) varieties. Amer. Bee J., 125 (5), 351-354.

TRAYNOR J., 1981. - Use of a fast and accurate method for evaluating pollen production of alfalfa and almond flowers, Amer. Bee J., 121 (1), 23-25.

WarakomsKa Z., 1972. - Etude sur la production du pollen provenant de la flore (en polonais). Pscz. Zesz. Nauk., 16, 63-90. 